Proceedings of the 47th International School and Conference on the Physics of Semiconductors "Jaszowiec 2018"

\title{
Solvent Driven Magnetic Order Change in Graphene Decorated with $\alpha-\mathrm{Fe}_{2} \mathrm{O}_{3}$ Nanoparticles
}

\author{
L. Kilanski ${ }^{a}$, M. Arciszewska ${ }^{a}$, R. Jedrzejewski ${ }^{b}$, D. SiberA ${ }^{c}$ And A. JedrzejewskA ${ }^{c, *}$ \\ ${ }^{a}$ Institute of Physics, Polish Academy of Sciences, Aleja Lotnikow 32/46, PL-02668 Warsaw, Poland \\ ${ }^{b}$ Institute of Materials Science and Engineering, Faculty of Mechanical Engineering and Mechatronics, \\ West Pomeranian University of Technology, Szczecin, Poland
}

${ }^{c}$ Institute of Chemical and Environment Engineering, West Pomeranian University of Technology, Szczecin, Poland

\begin{abstract}
We present the studies of the structural and magnetic properties of nanocomposites based on graphene decorated with $25 \% \alpha-\mathrm{Fe}_{2} \mathrm{O}_{3}$ nanoparticles made with the use of either ethanol or acetone. We observed the presence of superparamagnetic blocking of the $\alpha-\mathrm{Fe}_{2} \mathrm{O}_{3}$ nanoparticles at blocking temperature, $T_{B}=46.4 \mathrm{~K}$ or $159 \mathrm{~K}$, for the samples made with the use of either ethanol or acetone, respectively. Our magnetization and magnetic susceptibility data revealed the presence of strong inter-particle interactions in our samples. The magnetic properties of our samples depend strongly on the type of solvent used for the sample preparation.
\end{abstract}

DOI: 10.12693/APhysPolA.134.954

PACS/topics: gaphene, composites, magnetic properties

\section{Introduction}

Low-dimensional carbon related nanostructures [1], in particular graphene, became a subject of intense research due to their remarkable properties $[2,3]$. Decoration of graphene surface with magnetic nanoparticles is one of the major scientific topics in the recent years due to the high specific surface area of graphene [4] leading to the possibility of introducing new or improving the functional properties of base magnetic material. Nanocomposites based on graphene decorated with magnetic iron oxide nanoparticles show a variety of interesting properties prospective for applications in biomedicine, magnetic fluids, magnetic energy storage, environmental remediation, and catalysis $[5,6]$. Graphene decoration with transition metal oxides such as $\mathrm{Fe}_{2} \mathrm{O}_{3}[7]$ is expected to provide properties important from the point of view of applications due to low toxicity and biocompatibility of this iron oxide.

Our current paper is focused over the studies of structural and magnetic properties of composites prepared from commercial graphene with $25 \% \alpha-\mathrm{Fe}_{2} \mathrm{O}_{3}$ made with the use of either ethanol or acetone solvent. In particular, we wish to study the influence of the solvent type on the magnetic properties of the composite.

\section{Sample preparation}

For the preparation of composite materials, graphene obtained using the Hummers method and iron nitrate(III) was used. Graphene was first dispersed in ethanol or in acetone. An appropriate quantity of iron nitrate (to reach $25 \mathrm{wt} \%$ of $\mathrm{Fe}_{2} \mathrm{O}_{3}$ in the composite) was

\footnotetext{
* corresponding author; e-mail:

Anna. Jedrzejewska@zut . edu.pl
}

weighted into a beaker and $50 \mathrm{ml}$ of ethanol or acetone was added. Graphene in ethanol or acetone was sonificated for $10 \mathrm{~min}$. A volume of $0.5 \mathrm{ml}$ of the suspension (approximately $0.2 \mathrm{~g}$ of graphene) was then transferred to the solution with iron nitrate and stirred for $10 \mathrm{~min}$. Then, ammonia water was added to the slurry to reach pH 11 and to precipitate the iron hydroxide. The reaction mixture was next transferred to a Teflon container and placed in an autoclave. Composite materials were obtained in an autoclave at a temperature of $120^{\circ} \mathrm{C}$ kept for $12 \mathrm{~h}$. The obtained composites were rinsed with distilled water and dried at $80^{\circ} \mathrm{C}$ for $48 \mathrm{~h}$.

\section{Structural characterization}

The XRD pattern of prepared samples are presented in Fig. 1. We studied the structural quality using the Empyrean (PANalytical) X-ray diffractometer with the use of $\mathrm{Cu} K_{\alpha}(\lambda=1.54 \AA)$.

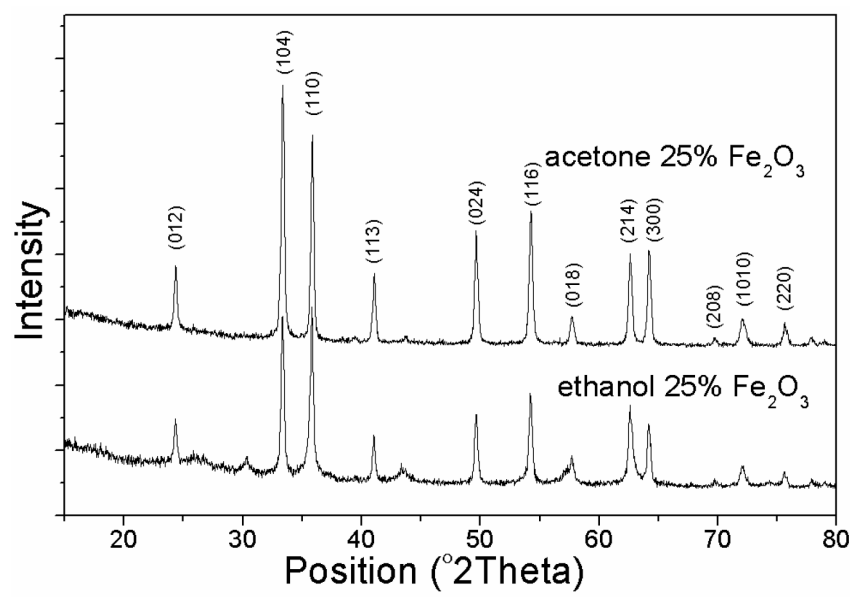

Fig. 1. Diffraction patterns obtained for the graphene $+\mathrm{Fe}_{2} \mathrm{O}_{3}$ samples prepared with the use of acetone or ethanol. 


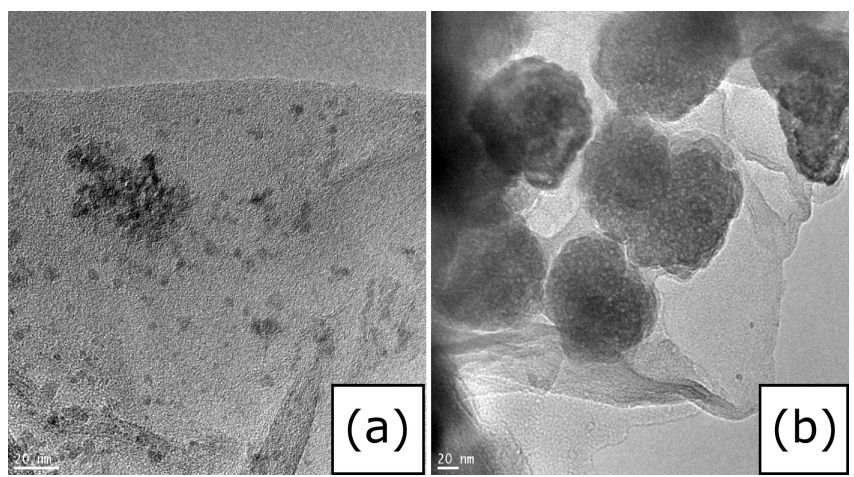

Fig. 2. TEM images of composite obtained in (a) ethanol and (b) acetone.

All peaks on the XRD patterns can be assigned to the rhombohedral $\mathrm{Fe}_{2} \mathrm{O}_{3}$ phase (ICDD 01-072-0469, space group $R-3 c$, space group number $167, h k l$ inserted on the graph).

The attached pictures (see Fig. 2) show that the reaction environment affects the morphology of the obtained composites. In the sample obtained in acetone, it can be seen that $\mathrm{Fe}_{2} \mathrm{O}_{3}$ forms agglomerates with a size above $100 \mathrm{~nm}$. Agglomerates are also observed in the sample obtained in ethanol, but in this case they are smaller. In addition, the size of crystallites in the sample obtained in ethanol is smaller - below $10 \mathrm{~nm}$, and the $\mathrm{Fe}_{2} \mathrm{O}_{3}$ crystals are more evenly distributed on the surface of graphene in that sample.

\section{Magnetic properties}

We made detailed measurements of the magnetic properties of two graphene $+25 \% \alpha-\mathrm{Fe}_{2} \mathrm{O}_{3}$ samples made with either ethanol or acetone with the use of the LakeShore 7229 Susceptometer/Magnetometer system. Our studies consisted of the measurements of the dynamic magnetic susceptibility, $\chi_{A C}$, and magnetization, $M$, done at temperatures from $T=4.3 \mathrm{~K}$ up to $320 \mathrm{~K}$. The AC magnetic susceptibility measurements were done using mutual inductance method. The static magnetization measurements done using the Weiss extraction technique were made at $B \leq 9 \mathrm{~T}$ and at temperatures from $T=4.3 \mathrm{~K}$ up to $320 \mathrm{~K}$. Magnetization measurements were also done with the use of the alternating gradient magnetometer (AGM).

The ac magnetic susceptibility was studied as a function of temperature for both samples containing composites made with either ethanol or acetone. The results of the measurements in the form of the temperature dependence of the real part of the magnetic susceptibility, $\operatorname{Re}\left(\chi_{A C}\right)(T)$, is presented in Fig. 3 .

As we can see in Fig. 3, we observe large differences in the $\operatorname{Re}\left(\chi_{A C}\right)(T)$ dependences between the two studied samples. The $\operatorname{Re}\left(\chi_{A C}\right)(T)$ dependences for our two samples show values order of magnitude higher than the magnetic susceptibility observed for pure graphene samples, being the reference for us and being the subject

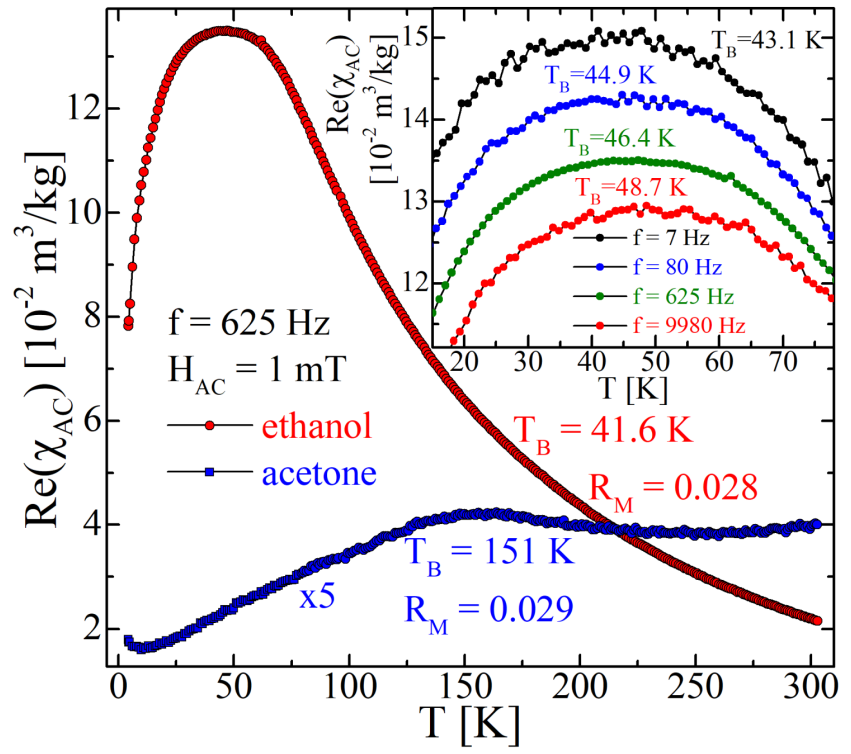

Fig. 3. Temperature dependence of the real part of the magnetic susceptibility obtained for two studied graphene $+25 \% \alpha-\mathrm{Fe}_{2} \mathrm{O}_{3}$ samples. The values of the $\operatorname{Re}\left(\chi_{A C}\right)(T)$ dependence for the sample made with the use of acetone are multiplied by 5 . The inset shows frequency shift of the $\operatorname{Re}\left(\chi_{A C}\right)(T)$ dependence for the sample made with the use of ethanol.

of our studies in Ref. [8]. It is clear signature that the magnetic properties of the graphene $+\alpha-\mathrm{Fe}_{2} \mathrm{O}_{3}$ composite are dominated by the component related to $\alpha$ $\mathrm{Fe}_{2} \mathrm{O}_{3}$ nanoparticles and depend strongly on the solvent used during the sample synthesis. At $T<20 \mathrm{~K}$, for the sample made with the use of acetone, we observed a small decrease in the $\operatorname{Re}\left(\chi_{A C}\right)(T)$ dependence, a feature not observed for the sample made with the use of ethanol, and related to the presence of isolated paramagnetic magnetic moments in this sample. The $\operatorname{Re}\left(\chi_{A C}\right)(T)$ dependence obtained for our sample made with the use of ethanol shows the presence of a well-defined maximum at temperature of about $45 \mathrm{~K}$. On the other hand, for the sample made with the use of acetone, the observed $\operatorname{Re}\left(\chi_{A C}\right)(T)$ dependence shows values an order of magnitude lower and the presence of a cusp at temperature of about $150 \mathrm{~K}$ followed with an increasing dependence at room temperature. The presence of a symmetric maximum in the $\operatorname{Re}\left(\chi_{A C}\right)(T)$ curve indicates the presence of a magnetic transition in the system. Identification of the type of the observed transition requires more detailed magnetometric measurements and data analysis.

We made $\operatorname{Re}\left(\chi_{A C}\right)(T)$ measurements for four different frequencies of the AC magnetic field, $f=7,80$, 625 , and $9980 \mathrm{~Hz}$. The results obtained for the selected graphene $+25 \% \alpha-\mathrm{Fe}_{2} \mathrm{O}_{3}$ sample are presented in the inset to Fig. 3. The maxima of the $\operatorname{Re}\left(\chi_{A C}\right)(T)$ curves obtained at different magnetic field frequencies shift on the temperature scale with an increase of $f$ for both our samples. The position at the temperature scale of the maxima in the $\operatorname{Re}\left(\chi_{A C}\right)(T)$ dependence defines the spe- 
cific blocking temperature, $T_{B}$. The $T_{B}(f)$ dependence can be analyzed using the phenomenological parameter, $R_{M}$, proposed by Mydosh [9] defined with the following formula:

$$
R_{M}=\frac{\Delta T_{B}}{\left(T_{B}\right) \Delta \log (f)},
$$

where $\Delta T_{B}=T_{B}\left(f_{i}\right)-T_{B}\left(f_{j}\right)$, and $\Delta \log (f)=\log \left(f_{i}\right)-$ $\log \left(f_{j}\right)$. For both our samples we obtained similar $R_{M}$ values about 0.03 . The $R_{M}$ values should be around 0.01 for spin-glasses while $R_{M}>0.1$ is known for superparamagnetic systems [9]. The $R_{M}$ values calculated for our samples are smaller than the values known for the case of the superparamagnetic $\alpha-\mathrm{Fe}_{2} \mathrm{O}_{3}$ nanoparticles with $R_{M}>0.1$. It is a signature of the presence of strong inter-particle interactions between the $\alpha$ - $\mathrm{Fe}_{2} \mathrm{O}_{3}$ nanoparticles [10] present in our samples causing the system to behave like a spin-glass.

The experimental $T_{B}(f)$ dependences allowed us to estimate the values of the static true superparamagnetic blocking temperature, $T_{B}$. We approximated the $T_{B}(f)$ dependence to $f \rightarrow 0 \mathrm{~Hz}$ to estimate $T_{B}$ values of our samples. The estimated $T_{B}$ values for our samples are gathered in Fig. 3. The temperature of superparamagnetic blocking of the $\alpha-\mathrm{Fe}_{2} \mathrm{O}_{3}$ nanoparticles changes with the average nanoparticle size and the type of the host matrix. Literature data shows that $T_{B}$ values changes from $19 \mathrm{~K}$ for $4 \mathrm{~nm}$ diameter nanoparticles in the silica matrix [11], $T_{B} \approx 22 \mathrm{~K}$ for $5 \mathrm{~nm}$ diameter nanoparticles embedded in the polymer matrix [12] up to $T_{B} \approx 120 \mathrm{~K}$ for the $3 \mathrm{~nm}$ diameter nanoparticles embedded in the alumina matrix [13]. The differences in $T_{B}$ observed for our samples are related to the change of the average diameter of the nanoparticles between the samples made with the use of either ethanol or acetone.

For the sample made with the use of acetone we observed signatures of the presence of another magnetic transition at $T>320 \mathrm{~K}$, outside of the measurement range. It is probable that this transition will be the Morin transition in this sample, a feature observed for the $\alpha-\mathrm{Fe}_{2} \mathrm{O}_{3}$ systems [14].

We made the magnetization measurements as a function of the applied static magnetic field $B \leq 9 \mathrm{~T}$ at several stabilized temperatures lower than $T=200 \mathrm{~K}$. The selected $M(B)$ curves obtained for our samples are presented in Fig. 4.

The $M(B)$ curve obtained for our graphene $+25 \%$ $\alpha-\mathrm{Fe}_{2} \mathrm{O}_{3}$ samples varies between the samples. Both magnetization curves presented in Fig. 4 shows lack of saturation even at $B=9 \mathrm{~T}$. It is a signature of a strong magnetic frustration in the system, most probably related to the presence of strong inter-particle interactions in our samples. We observe large differences in the magnetization values observed for both our samples. This result, in agreement with the magnetic susceptibility data, indicates the significance of the preparation procedure in the effective magnetic moment of the graphene $+\alpha-\mathrm{Fe}_{2} \mathrm{O}_{3}$ nanocomposites.

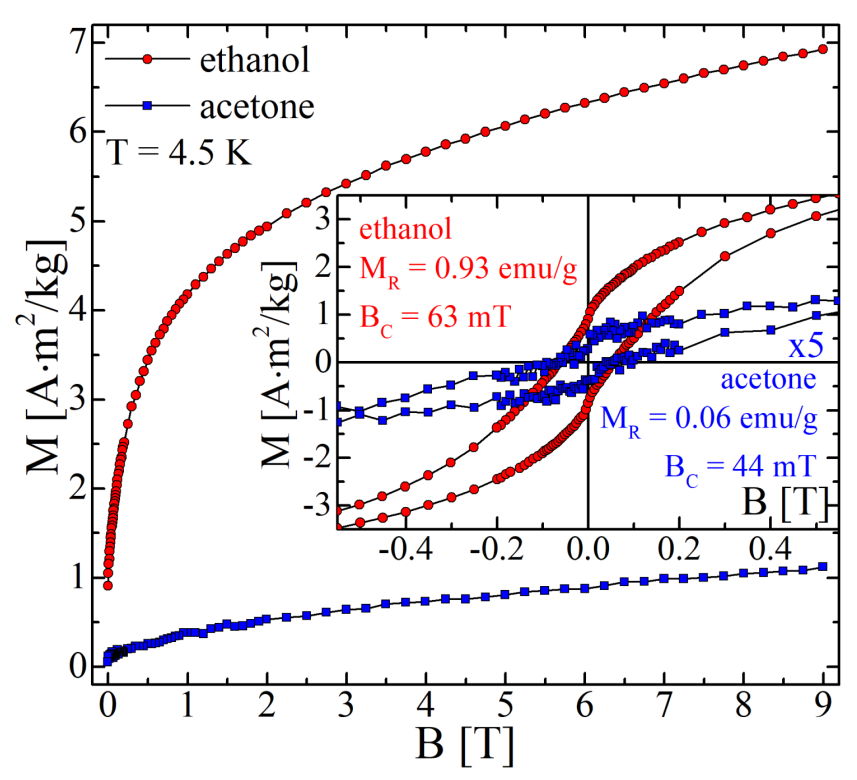

Fig. 4. Magnetic field dependence of the magnetization obtained for the graphene $+25 \% \alpha-\mathrm{Fe}_{2} \mathrm{O}_{3}$ composite samples made with the use of either ethanol or acetone. In the inset, the values of the $M(B)$ dependence for the sample made with the use of acetone are multiplied by 5 .

We also performed the $M(B)$ hysteresis curves measurements at $T<300 \mathrm{~K}$ for both our samples. The obtained $M(B)$ hysteresis curves obtained at $T \approx 4.5 \mathrm{~K}$ for our samples are presented in the inset to Fig. 4 . The irreversible $M(B)$ curves behavior is present for all our graphene $+25 \% \alpha-\mathrm{Fe}_{2} \mathrm{O}_{3}$ samples. Inspection of data in the inset to Fig. 4 shows difference in the $M(B)$ hysteresis curves between the samples made with the use of ether acetone or ethanol. Both $M(B)$ hysteresis curves have elliptical shape indicating the existence of distribution of magnetic domain structure of the $\alpha-\mathrm{Fe}_{2} \mathrm{O}_{3}$ nanoclusters.

We calculated two parameters describing the shape of the $M(B)$ hysteresis curves for our samples, e.g., the remnant magnetization and coercive field, $M_{R}$ and $B_{C}$, respectively. Both the $M_{R}(T)$ and the $B_{C}(T)$ dependences for our samples decrease as a function of temperature and at $T>T_{B}$ our samples does not show magnetic irreversibility. Both $M_{R}$ and $B_{C}$ values are higher for the sample made with the use of ethanol than the values obtained for the sample made with the use of acetone (see labels to the inset to Fig. 4). It is therefore possible to tune the magnetic properties of the nanocomposite via preparation procedure.

\section{Summary}

Summarizing, we report the experimental studies of the magnetic properties of two graphene $+25 \% \alpha-\mathrm{Fe}_{2} \mathrm{O}_{3}$ nanocomposites made with the use of either ethanol or acetone. The magnetic properties of the studied composites strongly depend on the type of the solvent used 
during the sample synthesis. For both our samples we observe the presence of superparamagnetic blocking at temperatures $T_{B} \approx 50 \mathrm{~K}$ and $150 \mathrm{~K}$ for the sample made with the use of ethanol or acetone, respectively. The superparamagnetic blocking, however, is suppressed by the presence of strong inter-particle interactions. The change of the synthesis solvent plays major role in the magnetic behavior of our samples resulting in much higher magnetization for the sample made with the use of ethanol. Both the shape and the parameters characterizing the magnetization hysteresis loops depend strongly on the change of the preparation method. We clearly show the possibilities to control the magnetic properties of graphene $+\mathrm{Fe}_{2} \mathrm{O}_{3}$ nanocomposite over a wide range of values.

\section{Acknowledgments}

This work was supported by project: LIDER/496/L6/14/NCBR/2015 financed by The National Centre for Research and Development.

\section{References}

[1] P. Ehrenfreund, B.H. Foing, Science 329, 1159 (2010).

[2] C. Reeves, Graphene: Characterization After Mechanical Exfoliation 2010.
[3] S. Stankovich, D.A. Dikin, G.H.B. Dommett, K.M. Kohlhaas, E.J. Zimney, E.A. Stach, R.D. Piner, S.T. Nguyen, R.S. Ruoff, Nature 442, 282 (2006).

[4] X. Ma, H. Tao, K. Yang, L. Feng, L. Cheng, X. Shi, Y. Li, L. Guo, Z. Liu, Nano Res. 5, 199 (2012).

[5] A.H. Lu, E.L. Salabas, F. Schuth, Angew. Chem. Int. Ed. 46, 1222 (2007).

[6] N.A. Frey, S. Peng, K. Cheng, S.H. Sun, Chem. Soc. Rev. 38, 2532 (2009).

[7] H.P. Cong, J.J. He, Y. Lu, S.H. Yu, Small 6, 169 (2009).

[8] A. Jedrzejewska, L. Kilanski, D. Sibera, S. Lewińska, A. Ślawska-Waniewska, P.S. Wrobel, A. Bachmatiuk, R. Jedrzejewski, J. Magn. Magn. Mater. 471, 321 (2019).

[9] J.A. Mydosh, Spin Glasses: An Experimental Introduction, Taylor and Francis, London 1994.

[10] M.F. Hansen, C. Bender Koch, S. Mørup, Phys. Rev. B 621124 (2000).

[11] M. Tadić, D, Marković, V. Spasojević, V. Kusigerski, M. Remškar, J. Pirnat, Z. Jagličić, J. Alloys Comp. 441, 291 (2007)

[12] R.D. Zysler, M. Vasquez Mansilla, D. Fiorani, Eur. Phys. J. B 41, 171 (2004).

[13] R.D. Zysler, D. Fiorani, A.M. Testa, J. Magn. Magn. Mater. 224, 5 (2001).

[14] R.N. Bhowmik, A. Saravanan, J. Appl. Phys. 107 053916 (2010). 